\title{
Radiative transfer simulation of water rotational excitation in comets
}

\section{Comparison of the Monte Carlo and escape probability methods ${ }^{\star}$}

\author{
V. Zakharov, D. Bockelée-Morvan, N. Biver, J. Crovisier, and A. Lecacheux
}

\begin{abstract}
LESIA, Observatoire de Paris - Section de Meudon, 5 place Jules Janssen, 92195 Meudon, France
e-mail: zvv1661@yandex.ru
\end{abstract}

Received 8 November 2006 / Accepted 4 June 2007

ABSTRACT

\begin{abstract}
Context. The recent advent of space-based detectors at far-infrared and submillimetre wavelengths opened up the possibility of observing cometary water from its rotational transitions. The $1_{10}-1_{01}$ fundamental line of ortho $\mathrm{H}_{2} \mathrm{O}$ at $557 \mathrm{GHz}$ was detected in several comets by the Submillimeter Wave Astronomical Satellite and Odin. This line as well as other water and $\mathrm{H}_{2}^{18} \mathrm{O}$ lines will be observed by the three instruments of the ESA Herschel Space Observatory. In order to prepare or interpret these observations, excitation models including $\mathrm{H}_{2} \mathrm{O}$ self-absorption are required.

Aims. For treating radiation transfer of water in comets, Bockelée-Morvan (1987, A\&A, 181, 169) used the local approximation with the escape probability method (EP). Bensch \& Bergin (2004, ApJ, 615, 531) used the Monte-Carlo method (MC), which is more exact from a physical point of view, but is much more CPU time-demanding. The aim of this study is to compare the results of the two methods and to investigate the extent to which the EP method provides acceptable results for synthesizing line profiles and analysing observations.

Methods. We developed two 1D numerical codes. The MC code is based on the accelerated Monte Carlo algorithm proposed by Hogerheijde \& van der Tak (2000, A\&A, 362, 697). The EP code is based on the algorithm proposed by Bockelée-Morvan (1987). They include the seven lowest rotational levels of ortho-water, which are the primarily populated levels in the rotationally cold coma. A spherically symmetric density distribution with constant expansion velocity is assumed. Collisions with water and electrons, and infrared pumping, are taken into account. Synthetic line profiles pertaining to Odin and future Herschel observations with the Heterodyne Instrument for Far-Infrared are computed for water production rates ranging from $10^{28}$ to $10^{30} \mathrm{~s}^{-1}$.

Results. We show that the EP method has sufficient accuracy to predict rotational excitation, line intensities and line shapes. Differences in level populations do not exceed $20 \%$, except in the narrow region where a strong gradient in electron temperature and density is present. Line shapes are in excellent agreement and line areas differ by less than $7 \%$.
\end{abstract}

Key words. comets: general - radio lines: solar system - submillimeter - radiative transfer - line: formation - methods: numerical

\section{Introduction}

Because water is the main constituent of cometary nucleus ices, its observation in cometary atmospheres is of paramount importance to measure the outgassing rate, monitor overall comet activity and determine relative abundances of minor gaseous constituents for chemical comparisons between comets. Water has been investigated by various means, either directly or indirectly (see reviews of Bockelée-Morvan et al. 2004; Feldman et al. 2004, and references therein).

The recent advent of space-based detectors at far-infrared and submillimetre wavelengths opened a new window for these investigations. Several rotational water lines were detected in comet C/1995 O1 (Hale-Bopp) with the Long Wavelength Spectrometer (LWS) of the Infrared Space Observatory (ISO) (Crovisier et al. 1997). The Submillimeter Wave Astronomical Satellite (SWAS, Melnick et al. 2000), a 60-cm telescope launched in 1998, provided the first detection of the $\mathrm{H}_{2} \mathrm{O} 1_{10}-1_{01}$ fundamental line at $556.9 \mathrm{GHz}$ in comet $\mathrm{C} / 1999 \mathrm{H} 1$ (Lee) (Neufeld et al. 2000). The Odin satellite, launched in 2001 (Nordh et al. 2003), observed with its 1-m antenna the same line in a dozen comets at high spectral resolution and also

* Figures 1, 2, 4, 7,9 and Table 3 are only available in electronic form at http://www. aanda.org detected the $1_{10}-1_{01}$ line at $547.7 \mathrm{GHz}$ of the $\mathrm{H}_{2}^{18} \mathrm{O}$ isotopologue in a few comets (Lecacheux et al. 2003; Biver et al. 2007). The Herschel Space Observatory, equipped with a $3.5-\mathrm{m}$ diameter cooled telescope, will cover the 60-600 $\mu \mathrm{m}$ range which contains many rotational lines of water (Pilbratt 2005). The observation of $\mathrm{H}_{2} \mathrm{O}$, HDO and $\mathrm{H}_{2}^{18} \mathrm{O}$ in cometary atmospheres is among the highest priorities of Solar System studies with Herschel (Crovisier et al. 2005). The Millimeter Instrument for the Rosetta Orbiter (MIRO) has been designed to observe the $1_{01}-1_{10}$ lines of $\mathrm{H}_{2} \mathrm{O}, \mathrm{H}_{2}^{17} \mathrm{O}$ and $\mathrm{H}_{2}^{18} \mathrm{O}$ for the space exploration of comet 67P/Churyumov-Gerasimenko (Gulkis et al. 2007).

Several models have been developed to prepare and interpret observations of water rotational lines in cometary atmospheres. As detailed by Crovisier (1984) and Weaver \& Mumma (1984), the main excitation mecanisms of the $\mathrm{H}_{2} \mathrm{O}$ molecule are collisional excitation and radiative pumping of the fundamental bands of vibration by the solar infrared flux. The former mechanism dominates in the inner coma and leads to thermal equilibrium. The latter conducts to a rotationally cold fluorescence equilibrium in the outer coma. Optical depth effects cannot be neglected. Bockelée-Morvan (1987) developed a 1D model of water excitation for spherically symmetric atmospheres in which $\mathrm{H}_{2} \mathrm{O}-\mathrm{H}_{2} \mathrm{O}$ collisions and vibrational excitation are included, and where radiation transfer is treated with the local approximation 
using the escape probability method (Ribicki 1984). The model was improved to include $\mathrm{H}_{2} \mathrm{O}$-electron collisional excitation (Biver 1997) and the infrared excitation of additional vibrational bands (Chiu et al. 2001). This model is currently used to analyse the Odin cometary data (Biver et al. 2007).

Bensch \& Bergin (2004) presented a 1D numerical model of water excitation which includes the same excitation processes, but where radiation transfer is rigorously solved using the accelerated Monte Carlo code developed by Hogerheijde \& van der Tak (2000). Indeed, the escape probability (EP) approach, which is much more computationally efficient in terms of CPU time, was implemented for specific physical conditions, namely for interstellar and circumstellar clouds with large velocity gradients and physical properties that do not vary over the distance where the Doppler shift is less than the line width. In the case of cometary atmospheres, the $\mathrm{H}_{2} \mathrm{O}$ molecules expand with a practically constant velocity which does not exceed the thermal velocity by more than a factor of about 5 . Velocity gradient is present only due to the geometry of expansion. Though practical calculations showed good agreement between the EP method and more exact computations even in media violating the required conditions of applicability of the EP method (e.g., Bastian et al. 1980), the errors introduced when treating cometary atmospheres have not been yet investigated.

We have developed a numerical code for the simulation of ortho-water excitation in cometary coma, based on the accelerated Monte Carlo (MC) algorithm (Hogerheijde \& van der Tak 2000). The model, in its $1 \mathrm{D}$ version, is therefore very similar to that developed by Bensch \& Bergin (2004). The main goal of this paper is to compare the results of the 1D model to those provided by the EP method. Rotational populations and emergent line profiles for observations with Odin and the Heterodyne Instrument for Far-Infrared (HIFI, de Graauw \& Helmich 2001) on Herschel are compared in Sect. 4. Comet model parameters were chosen close to typical objects that can be observed with Odin and Herschel. Results for comets of different activity are presented.

\section{Computational methods}

The radiative transfer equation can be written as

$\frac{\mathrm{d} I_{v}}{\mathrm{~d} \tau_{v}}=I_{v}-S_{v}$.

Here, $I_{v}$ is the specific intensity $\left[\mathrm{J} \mathrm{m}^{-2} \mathrm{sr}^{-1}\right]$ at frequency $v[\mathrm{~Hz}]$, $S_{v} \equiv j_{v} / \alpha_{v}$ is the source function ( $\alpha_{v}$ is the line absorption coefficient $\left[\mathrm{m}^{-1}\right], j_{v}$ is the emission coefficient $\left[\mathrm{J} \mathrm{m}^{-3} \mathrm{sr}^{-1}\right]$ ) and $\mathrm{d} \tau_{v} \equiv \alpha_{v} \mathrm{~d} s$ is the optical thickness along particular line of sight parameterized by $\mathrm{d} s$.

The average intensity $J_{v}\left[\mathrm{~J} \mathrm{~m}^{-2}\right]$ received from all solid angles $\mathrm{d} \Omega$ is:

$J_{v}=\frac{1}{4 \pi} \int I_{\nu} \mathrm{d} \Omega$.

Here, $I_{v}$ is the solution of Eq. (1) along each direction under consideration.

Knowing $J_{v}$ and assuming statistical equilibrium, the level populations can be determined from balance equations:

$$
\begin{aligned}
& n_{i}\left[\sum_{j<i} A_{i j}+\sum_{j \neq i}\left(B_{i j} J_{v}+C_{i j}\right)\right]= \\
& \sum_{j>i} n_{j} A_{j i}+\sum_{j \neq i} n_{j}\left(B_{j i} J_{v}+C_{j i}\right) .
\end{aligned}
$$

Here, $A_{i j}\left[\mathrm{~s}^{-1}\right]$ is the Einstein A-coefficient for spontaneous emission $(i \rightarrow j), B_{j i}$ and $B_{i j}\left[\mathrm{~J}^{-1} \mathrm{~m}^{2} \mathrm{~s}^{-1}\right]$ are the transition probabilities for absorption and stimulated emission, $C_{i j}$ is the collision rate $\left[\mathrm{s}^{-1}\right]$.

Starting with an initial guess for the level populations, $J_{v}$ is calculated, statistical equilibrium is solved and new level populations are obtained; the new populations yield a new value for $J_{v}$, after which the populations are updated, etc., until the radiation field and the populations have converged to a consistent solution. To compute $J_{v}$, direct numerical integration of Eq. (2) is possible, but it needs enormous computational time. Thus several methods are proposed for its solution.

\subsection{Monte Carlo method}

In the Monte Carlo method the source is divided into a large number of cells with constant physical properties (density, velocity, temperature, etc.). It is assumed that the molecular excitation in the cell is uniform. For each cell $J_{v}$ (Eq. (2)) is approximated by the summation over a random set of rays which enter the cell from infinity (or the boundary with a given boundary condition) from a random direction and, after propagation through the intervening cells, contribute to the radiation field within the cell. Then Eq. (3) is solved through matrix inversion. As $J_{v}$ depends on the level populations, the problem is solved iteratively, and a new $J_{v}$ is evaluated on each iteration.

The MC method is quite precise from a physical point of view and very flexible with respect to geometry. However, it can be quite slow as it demands that a large number of rays from each cell to follow through the source.

The Monte Carlo code that we have elaborated follows the accelerated Monte Carlo algorithm presented in Hogerheijde \& van der Tak (2000).

\subsection{Escape probability (Sobolev's) method}

The EP method reduces the global problem to a local one by assuming that each point of the gas is essentially coupled radiatively with the near region surrounding it. For systematic velocity fields, the essence of this approximation lies in the fact that, if along the path of a line photon velocity gradients are large compared to the thermal velocity $v_{\text {th }}$ characterizing the line width, there is a local region within which the photon has a finite probability of being absorbed in the line and beyond which a vanishing probability of doing so.

The escape probability method assumes that $J_{v}$ could be expressed as:

$J_{v}=\left(1-\beta_{v}\right) S_{v}+\beta_{v}^{c} I_{v}$.

$\beta_{v}$ is the average over $4 \pi$ solid angle of $\beta_{v}(\boldsymbol{n})$ which is the probability that an emitted photon escapes the line trapping region in direction $\boldsymbol{n}$ without suffering any absorption. $\beta_{v}^{c} I_{v}$ describes the amount of external radiation $I_{v}$ participating in the excitation. $\beta_{v}^{c}$ is $\beta_{v}(\boldsymbol{n})$ averaged over the solid angle of the excitation source.

For a spherically symmetric media expanding at constant velocity $v_{\text {exp }}$, which approximates cometary atmospheres, the escape probabilities are given by Litvak \& Kuiper (1982).

In the code which uses the EP method, the equations of statistical equilibrium are time-dependent and describe the evolution of level populations for the molecule moving in the coma (Bockelée-Morvan 1987). The system of ordinary differential equations is solved numerically by a method for stiff equations. This is in contrast with the Monte Carlo code where these equations correspond to the volume of the cell and are solved by 
matrix inversion assuming steady state. We found that the results obtained by the EP method do not depend on the numerical method used for solving the statistical equilibrium equations.

\section{Comet model}

In the following we only briefly discuss the physical model of the coma used in the present study. For the most part, the present model follows that proposed in Bensch \& Bergin (2004). An introduction to molecular excitation processes in cometary atmospheres can be found in Bockelée-Morvan et al. (2004).

We consider nine rotational transitions connecting the seven lowest rotational levels in the ground vibrational state of ortho- $\mathrm{H}_{2} \mathrm{O}$ (see Fig. 1).

\subsection{Water vapour atmosphere}

The distribution of the water density $n_{\mathrm{H}_{2} \mathrm{O}}\left[\mathrm{m}^{-3}\right]$ at the cometocentric distance $r[\mathrm{~m}]$ in the coma is represented by the standard Haser distribution (Haser 1957),

$n_{\mathrm{H}_{2} \mathrm{O}}(r)=\frac{Q_{\mathrm{H}_{2} \mathrm{O}}}{4 \pi r^{2} v_{\exp }} \exp \left(-r \frac{\beta_{\mathrm{H}_{2} \mathrm{O}}}{v_{\exp }}\right)$.

This model assumes isotropic outgassing at a constant rate from the nucleus and outflowing at a constant velocity $v_{\exp }\left[\mathrm{m} \mathrm{s}^{-1}\right]$. $Q_{\mathrm{H}_{2} \mathrm{O}}$ is the total water production rate from the nucleus $\left[\mathrm{s}^{-1}\right]$. The exponential term accounts for the finite lifetime of the water molecules due to photodissociation and ionization in the solar UV field, with total rate $\beta_{\mathrm{H}_{2} \mathrm{O}}\left[\mathrm{s}^{-1}\right]$.

Our codes can accommodate radial profiles of temperature and velocity. Nevertheless, for the model presented here, we assume a constant gas temperature $T_{\text {kin }}$ of the neutral species throughout the coma and a constant expansion velocity.

The radial density distribution is modelled from 10 to $10^{6} \mathrm{~km}$ using shells with constant physical properties. The width of the shells increases with the radius and the spacing is chosen to be equidistant in logarithmic space.

We assume an ortho-to-para ratio of 3 and thus an orthowater density profile of $n_{\mathrm{O}-\mathrm{H}_{2} \mathrm{O}}(r)=0.75 \times n_{\mathrm{H}_{2} \mathrm{O}}(r)$.

\subsection{Line excitation by $\mathrm{H}_{2} \mathrm{O}-\mathrm{H}_{2} \mathrm{O}$ collisions}

Excitation of $\mathrm{H}_{2} \mathrm{O}$ by collisions with other water molecules is important for inner regions with high gas densities. Collisions with other neutrals (e.g. CO, whose abundance may reach $20 \%$ that of water in some comets) may be significant but are not considered. The collision rates for de-excitation $C_{i j}^{\mathrm{H}_{2} \mathrm{O}-\mathrm{H}_{2} \mathrm{O}}\left[\mathrm{s}^{-1}\right]$ were computed using the $\mathrm{H}_{2} \mathrm{O}-\mathrm{H}_{2} \mathrm{O}$ cross-sections $\sigma_{i j}\left[\mathrm{~m}^{2}\right]$ calculated by Buffa et al. (2000) for the neutral gas temperature $T_{\text {kin }}=100 \mathrm{~K}$, as was done by Bensch \& Bergin (2004). The $C_{j i}^{\mathrm{H}_{2} \mathrm{O}-\mathrm{H}_{2} \mathrm{O}}$ are related to the $C_{i j}^{\mathrm{H}_{2} \mathrm{O}-\mathrm{H}_{2} \mathrm{O}}$ from the balance equation.

\subsection{Line excitation by $\mathrm{H}_{2} \mathrm{O}$-electron collisions}

The $\mathrm{H}_{2} \mathrm{O}$-electron collision rates $\left[\mathrm{s}^{-1}\right]$ are calculated following Biver (1997) (an extension of the work of Xie \& Mumma 1992). Here we assume that the velocity of the molecules is negligibly small in comparison with the velocity of electrons. Then, for deexcitation $(i \rightarrow j)$ :

$C_{i j}^{\mathrm{e}}=n_{\mathrm{e}}\left\langle v_{\mathrm{e}}\right\rangle \sigma_{i j}^{\mathrm{e}} 2 a_{i j} \exp \left(+a_{i j}\right) K_{0}\left(a_{i j}\right)$ and for excitation $(j \rightarrow i)$ :

$C_{j i}^{\mathrm{e}}=n_{\mathrm{e}}\left\langle v_{\mathrm{e}}\right\rangle \frac{g_{i}}{g_{j}} \sigma_{i j}^{\mathrm{e}} 2 a_{i j} \exp \left(-a_{i j}\right) K_{0}\left(a_{i j}\right)$

where $n_{\mathrm{e}}\left[\mathrm{m}^{-3}\right]$ is the density of electrons, $\left\langle v_{\mathrm{e}}\right\rangle$ is the averaged thermal speed of electrons $\left[\mathrm{m} \mathrm{s}^{-1}\right]$ and $g_{i}, g_{j}$ are the statistical weights of the levels labelled by $i, j . K_{0}$ is the Bessel function and $a_{i j}=h v_{i j} / 2 k T_{\mathrm{e}}$, where $T_{\mathrm{e}}[\mathrm{K}]$ is the electron temperature, $v_{i j}$ is the line central frequency [Hz] of the $i \rightarrow j$ transition.

The cross-sections $\left[\mathrm{m}^{2}\right]$ are derived from Itikawa's (1972) formulae and related to the Einstein-A coefficients for spontaneous emission $A_{i j}$ through :

$\sigma_{i j}^{\mathrm{e}}=\frac{m_{\mathrm{e}} e^{2} c^{3} A_{i j}}{16 \pi^{2} \varepsilon_{0} h^{2} v_{i j}^{4}}$,

where physical constants $h, e, c, \varepsilon_{0}$ and $m_{\mathrm{e}}$ have their usual meaning. Einstein-A coefficients and frequencies are taken from the GEISA molecular data base (Jacquinet-Husson et al. 1999).

For the electron density and temperature radial profiles, we used the same description as in Biver (1997). These profiles were derived from measurements in the coma of $1 \mathrm{P} /$ Halley by Giotto mass spectrometers applying physically justified scaling laws to account for their dependency with the water production rate and heliocentric distance of the comet.

The electron temperature $T_{\mathrm{e}}[\mathrm{K}]$ varies with distance to nucleus according to:

$T_{\mathrm{e}}(r)= \begin{cases}T_{\mathrm{kin}}, & r<R_{\mathrm{cs}} \\ T_{\mathrm{kin}}+\left(T_{\mathrm{e}}^{\mathrm{MAX}}-T_{\mathrm{kin}}\right)\left(\frac{r}{R_{\mathrm{cs}}}-1\right), & R_{\mathrm{cs}} \leq r \leq 2 R_{\mathrm{cs}} \\ T_{\mathrm{e}}^{\mathrm{MAX}}, & r>2 R_{\mathrm{cs}}\end{cases}$

where the distance of the contact surface $R_{\mathrm{cs}}[\mathrm{m}]$,

$R_{\mathrm{cs}}=\left(1.125 \times 10^{6}\right) x_{r_{\mathrm{e}}} Q_{29}^{3 / 4}$

includes the scaling factor $x_{r_{\mathrm{e}}}$ and $Q_{29}=Q_{\mathrm{H}_{2} \mathrm{O}} / 10^{29}$.

The electron density profile $n_{\mathrm{e}}\left[\mathrm{m}^{-3}\right]$ is approximated by:

$$
\begin{aligned}
n_{\mathrm{e}}(r)= & x_{n_{\mathrm{e}}}\left(\frac{Q_{\mathrm{H}_{2} \mathrm{O}} k_{\mathrm{ion}}}{v_{\mathrm{exp}} k_{\mathrm{rec}} r_{h}^{2}}\right)^{0.5}\left(\frac{T_{\mathrm{e}}}{300}\right)^{0.15}\left(\frac{R_{\mathrm{rec}}}{r^{2}}\right) \\
& \times\left[1-\exp \left(-\frac{r}{R_{\mathrm{rec}}}\right)\right]+\frac{5 \times 10^{6}}{r_{h}^{2}}
\end{aligned}
$$

where $R_{\text {rec }}=\left(3.2 \times 10^{6}\right) x_{r_{\mathrm{e}}} Q_{29}^{1 / 2}[\mathrm{~m}]$ is the recombination surface outside which the recombination rate of electrons with ions is insignificant. $k_{\text {ion }}\left[\mathrm{s}^{-1}\right]$ is the water photoionization rate at the heliocentric distance $r_{h}=1 \mathrm{AU}$ and $k_{\mathrm{rec}}=7 \times$ $10^{-13}\left(300 / T_{\mathrm{e}}\right)^{1 / 2}\left[\mathrm{~s}^{-1} \mathrm{~m}^{3}\right]$ is the recombination rate for electrons with $\mathrm{H}_{3} \mathrm{O}^{+}$ions. $x_{n_{\mathrm{e}}}$ is a scaling factor that is introduced to account for uncertainties in the modelling of the absolute electron density. The last term in Eq. (5) corresponds to the electron density in the solar wind.

These profiles with $x_{r_{\mathrm{e}}} \simeq 1$ and $x_{n_{\mathrm{e}}} \sim 1$ match the measurements performed in 1P/Halley onboard Giotto (Xie \& Mumma 1992). Mapping of the $\mathrm{H}_{2} \mathrm{O} 557 \mathrm{GHz}$ line with Odin suggests that $x_{n_{\mathrm{e}}}=0.2$ is more appropriate, however $x_{n_{\mathrm{e}}}=1$ will be used in this paper (Biver et al. 2007). An example of temperature and density profiles is shown in Fig. 2. 


\subsection{Line excitation by IR pumping and fluorescence}

Pumping of vibrational excited states by solar radiation and the subsequent rotational and vibrational decays contribute to the excitation of the rotational levels in the ground vibrational state. This process is mostly effective in the outer coma and results in a fluorescence equilibrium which depends only upon the heliocentric distance. Because rotational decay within the excited vibrational bands can be neglected, this process can be modelled by "effective" pumping rates $G_{i j}$ for the rotational levels in the ground vibrational state to be included in the statistical equilibrium equations. In other words, the vibrational excited states are not explicitly taken into account when solving for the population of the rotational levels within the ground vibrational state. In our computations we have neglected opacity effects which can affect ro-vibrational transitions. Indeed, they are only significant in the inner coma of comets with high production rates. This process is included in the model of Bensch \& Bergin (2004), using the escape probability formalism.

When collisions and radiation trapping are not considered, the equations of statistical equilibrium (Eq. (3)) then reduce to:

$n_{i}\left(\sum_{j<i} A_{i j}+\sum_{j \neq i} G_{i j}\right)=\sum_{j>i} n_{j} A_{j i}+\sum_{j \neq i} n_{j} G_{j i}$.

As described in Bockelée-Morvan \& Crovisier (1989), we considered excitation via the (001), (010), (100), (101) and (011) vibrational states and their subsequent cascades down to the (000) vibrational state, including via hot bands (e.g., (101) $\rightarrow(001)$ ). Pumping rates and Einstein-A coefficients of ro-vibrational transitions were computed using the GEISA molecular data bank (Jacquinet-Husson et al. 1999), as explained in BockeléeMorvan \& Crovisier (1989). Seven rotational states were considered in each excited state, as for the fundamental vibrational state. It is worth noting that the rates computed in this way are only approximate due to the small number of included levels (Fig. 1). This is particularly the case for the $3_{12}$ rotational level, whose population at fluorescence equilibrium at $r_{h}=1 \mathrm{AU}$ is underestimated by $30 \%$.

\section{Results}

The correctness of the code based on the MC algorithm was tested on the problem of $\mathrm{CO}$ line radiation transfer in a model of spherical, collapsing dark cloud studied by Bernes (1979).

In the present study, we compared the two methods in predicting two kinds of output data: level populations and synthetic line profiles. To measure the degree of agreement between results we introduce the relative difference $\delta x / x$ as follows:

$\frac{\delta x}{x}=\frac{\left|x_{\mathrm{MC}}-x_{\mathrm{EP}}\right|}{x_{\mathrm{EP}}}$

Here $x_{\mathrm{MC}}$ and $x_{\mathrm{EP}}$ are the values of any output parameter $x$ obtained by the MC and the EP methods, respectively.

\subsection{Parameters}

We consider comets with total gas production rate $Q_{\mathrm{H}_{2} \mathrm{O}}$ from $10^{28}$ to $10^{30} \mathrm{~s}^{-1}$ at a heliocentric distance $r_{h}=1 \mathrm{AU}$. The coma extends up to $10^{6} \mathrm{~km}$. Parameters for $r<1 \mathrm{~km}$ are those at $r=1 \mathrm{~km}$ (in order to exclude the singularity in the postulated law of density variation). In view of the size of the considered coma, this simplification gives small errors. Besides, we have
Table 1. Parameters of the model.

\begin{tabular}{l|c}
\hline \hline Parameter & \\
\hline Ortho-to-para ratio & 3 \\
Heliocentric distance, $r_{h}[\mathrm{AU}]$ & 1.0 \\
$\mathrm{H}_{2} \mathrm{O}$ photodissociation rate $\beta_{\mathrm{H}_{2} \mathrm{O}}\left[\mathrm{s}^{-1}\right]$ & $1.042 \times 10^{-5}$ \\
Expansion velocity, $v_{\mathrm{exp}}\left[\mathrm{km} \mathrm{s}^{-1}\right]$ & 0.85 \\
Neutral gas temperature, $T_{\mathrm{kin}}[\mathrm{K}]$ & 50.0 \\
Scaling factor contact surface, $x_{\mathrm{r}_{\mathrm{e}}}$ & 1.0 \\
Scaling factor electron density, $x_{n_{\mathrm{e}}}$ & 1.0 \\
Max. temperature of electrons, $T_{\mathrm{e}}^{\mathrm{MAX}}[\mathrm{K}]$ & $10^{4}$ \\
Photoionization rate $\left(r_{h}=1 \mathrm{AU}\right), k_{\mathrm{ion}}\left[\mathrm{s}^{-1}\right]$ & $4.1 \times 10^{-7}$ \\
\hline
\end{tabular}

Table 2. Parameters of computed cases.

\begin{tabular}{lccc}
\hline \hline Molec. & $Q_{\mathrm{H}_{2} \mathrm{O}}\left[\mathrm{s}^{-1}\right]$ & Abundance ratio & $\tau_{\mathrm{MAX}}^{*}$ \\
\hline $\mathrm{H}_{2} \mathrm{O}$ & $10^{30}$ & 1.0 & 361.0 \\
$\mathrm{H}_{2} \mathrm{O}$ & $10^{29}$ & 1.0 & 36.10 \\
$\mathrm{H}_{2} \mathrm{O}$ & $10^{28}$ & 1.0 & 3.610 \\
$\mathrm{H}_{2}^{18} \mathrm{O}$ & $10^{29}$ & 0.002 & 0.072 \\
\hline
\end{tabular}

${ }^{*}$ Opacity of $2_{12}-1_{01}$ line.

found it useful to compute the radiative excitation of $\mathrm{H}_{2}^{18} \mathrm{O}$ as an example of an optically thin coma. Input parameters common for all computations are listed in Table 1. Parameters specific to particular cases are listed in Table 2 . The value of $\tau_{\text {MAX }}$ listed in Table 2 is the opacity of the thickest line (namely the $2_{12}-1_{01}$ transition), computed along the radius from infinity to $r=1 \mathrm{~km}$. The values of $A_{i j}, \sigma_{i j}, G_{i j}$ and $v_{i j}$ used in the present study are given in Table 3. It should be noted that the parameters of the models are rather close to those considered in Bensch \& Bergin (2004).

\subsection{Convergency and accuracy of the MC computations}

In the radiative transfer calculations by the MC method, several specific factors affect the numerical accuracy:

1. The definition of convergence of the iterative procedure. The solution for level populations was considered to have converged when the noise-to-signal ratio in all cells for three subsequent iterations was less than $1 \%$. It should be noted that, due to strong differences in relative populations (up to $10^{3}$ between levels), small variations (e.g. due to statistical noise) in the population of one level, can cause relatively large variations for the other levels and noticeably decelerate convergence.

2. The grid convergence, i.e. dependence of results from the replacement of the integrals by finite sums (e.g. using a number of cells with constant properties in the formal solution of Eq. (1)). Grid convergence was checked by performing successive computations, increasing the number of cells. About 200 cells per decade were found sufficient to eliminate the dependency of the results on the cell size.

3. False convergence, i.e. when output values are rather far from the correct result, though the criterion of convergence is satisfied. The absence of false convergence was checked by several runs of the same case with different seeds of random number generator and different initial states. 

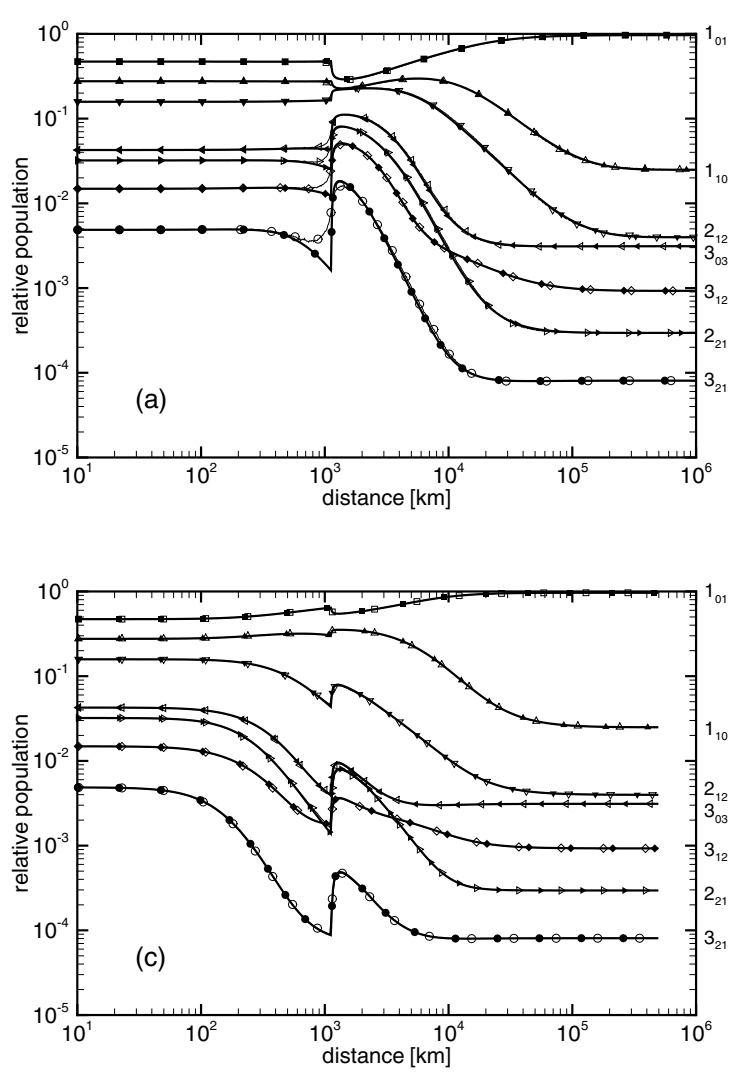

\subsection{Level populations}

Figures 3 and 4 show the ortho-water level populations obtained by the MC and the EP methods. The relative differences in level populations $\delta n / n$ for the case $Q_{\mathrm{H}_{2} \mathrm{O}}=10^{29} \mathrm{~s}^{-1}$ are shown in Fig. 5a. It is clearly visible that both methods are close $(\delta n / n<0.15)$ for most of the computational domain. Strong disagreement $(\delta n / n \sim 2-4)$ exists only in the vicinity of the contact surface $R_{\mathrm{cs}}$.

In the inner region, $\mathrm{H}_{2} \mathrm{O}-\mathrm{H}_{2} \mathrm{O}$ collisions dominate and the population distribution corresponds there to thermal equilibrium (LTE). Therefore the difference in definition of $J_{v}$ in the MC and the EP approaches makes no difference in the results and both methods agree very well $(\delta n / n<0.01)$. Let us recall that, in the EP method, thermal equilibrium was postulated on the inner boundary.

Beyond the LTE region starts the region where the neutral gas density is not high enough to maintain thermal equilibrium by only $\mathrm{H}_{2} \mathrm{O}-\mathrm{H}_{2} \mathrm{O}$ collisions. The $\mathrm{H}_{2} \mathrm{O}$-electron collisions and line trapping effects contribute also to the line excitation and adjust the excitation temperature to the local gas kinetic temperature. In this region at the distance $\sim R_{\mathrm{cs}}, \delta n / n$ rapidly increases up to a maximum $\sim 2-4$ (for higher levels). The reason for this divergence is that in contrast to the EP method, the MC method takes into account radiation coming from the nearby highly excited region at $r \geq R_{\mathrm{cs}}$ with enhanced electron temperature and number density. The EP method uses only local values of parameters. The extreme (maximal or minimal) values of level populations present at $\sim 2 R_{\mathrm{cs}}$ are in a good agreement (the difference between the two methods is less than $0.03-0.05$ for all levels except $3_{21}$ for which it is about $0.1-0.15$ ).

After passing the contact surface, results of both methods converge rapidly down to $\delta n / n<0.15$. In this region the MC

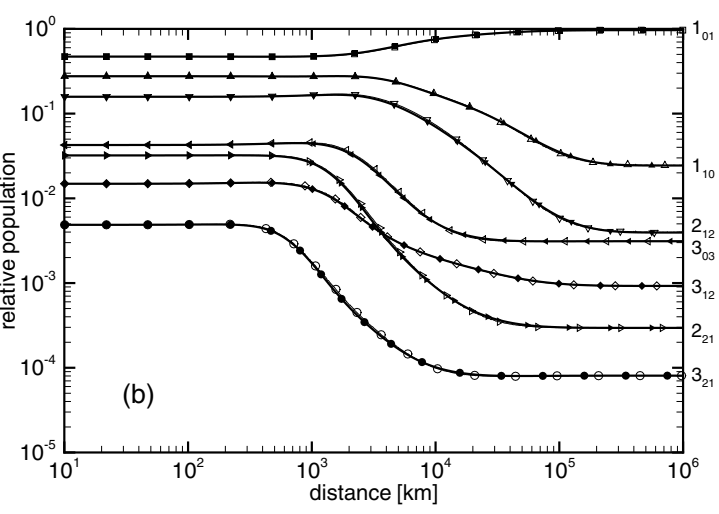

Fig. 3. Level populations of $\mathrm{H}_{2} \mathrm{O}$ by the $\mathrm{MC}$ (thin lines and open symbols) and the EP (thick lines and plain symbols) methods a) for $Q_{\mathrm{H}_{2} \mathrm{O}}=10^{29} \mathrm{~s}^{-1}$, b) for $Q_{\mathrm{H}_{2} \mathrm{O}}=$ $10^{29} \mathrm{~s}^{-1}$, case without $\mathrm{H}_{2} \mathrm{O}$-electron collisions, c) for $\mathrm{H}_{2}^{18} \mathrm{O}$ with $Q_{\mathrm{H}_{2} \mathrm{O}}=10^{29} \mathrm{~s}^{-1}$.

method takes into account radiation coming from the interior of the coma more precisely than the EP method (its influence decreases with the distance) and thus the relaxation distance of level populations appears to be longer in the MC method. In the outermost coma radiative transitions due to fluorescence start to dominate and the level populations gradually converge to fluorescence equilibrium. Since in both methods the same values of $G_{i j}$ are used, both methods agree very well again in this region $(\delta n / n<0.01)$. At large distances approximately $96.5 \%$ of the ortho-water molecules are in the ground rotational state $1_{01}$.

In the region where line trapping effects noticeably contribute to the line excitation, the EP method in general overestimates populations of the two lowest levels and underestimates populations of the higher levels. The most sensitive level is $3_{21}$ (the highest considered level). The important result found in this study is that the distributions of populations given by the two methods are very similar throughout the coma.

As an example of an optically thin case, we have considered radiative transfer for $\mathrm{H}_{2}^{18} \mathrm{O}$ in the atmosphere with an abundance ratio of $\mathrm{H}_{2}^{18} \mathrm{O}$ to $\mathrm{H}_{2} \mathrm{O}$ of $0.2 \%$ and $Q_{\mathrm{H}_{2} \mathrm{O}}=10^{29} \mathrm{~s}^{-1}$. As the evaluation of $J_{v}$ is unimportant, the results of both methods are practically identical (Fig. 3c).

In order to study the influence of the highly excited region (caused by the gradients in $T_{\mathrm{e}}$ and $n_{\mathrm{e}}$ ) we have computed the case without $\mathrm{H}_{2} \mathrm{O}$-electron collisions. The level populations obtained by the $\mathrm{MC}$ and the EP methods for the case $Q_{\mathrm{H}_{2} \mathrm{O}}=10^{29} \mathrm{~s}^{-1}$ are shown in Fig. 3b. The corresponding distribution of $\delta n / n$ is shown in Fig. 5b. As previously, we have good agreement $(\delta n / n \leq 0.01)$ in the inner and outer parts of the computational domain. In the central part the sharp peak at $\sim R_{\mathrm{cs}}$ has disappeared and we have $\delta n / n<0.1$, so there is a much closer agreement between the two methods compared to the previous case 

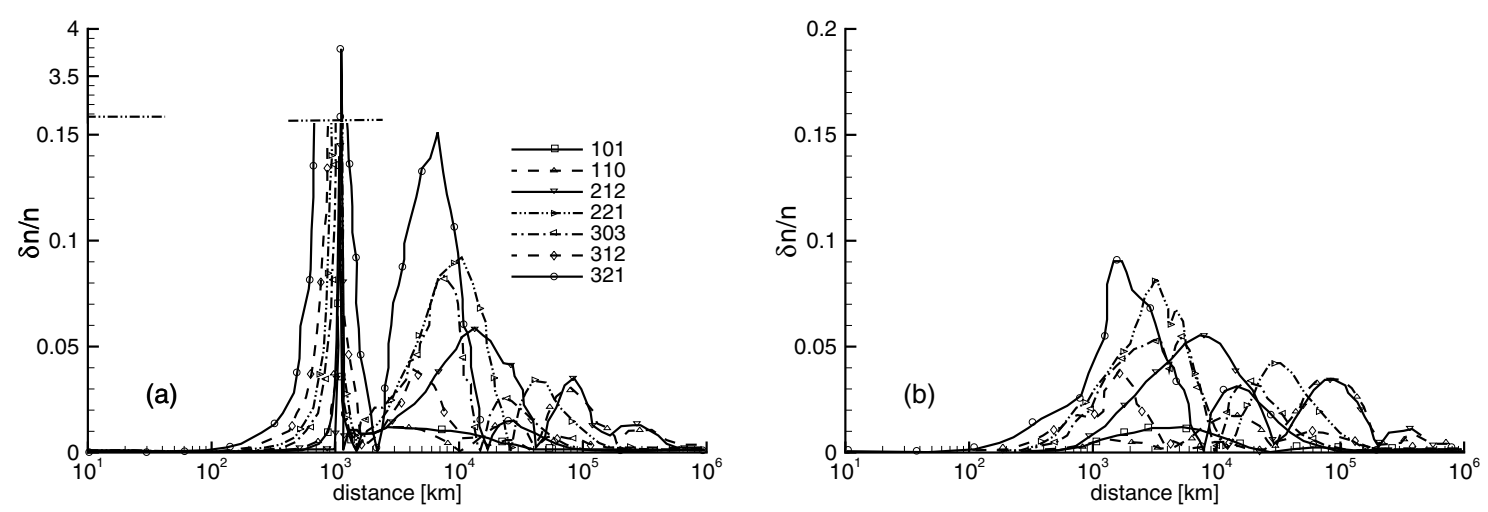

Fig. 5. Cometocentric variation of the relative differences in level populations $(\delta n / n)$ obtained with the EP and $\mathrm{MC}$ methods. a) for $Q_{\mathrm{H}_{2} \mathrm{O}}=10^{29} \mathrm{~s}^{-1}$, b) for $Q_{\mathrm{H}_{2} \mathrm{O}}=10^{29} \mathrm{~s}^{-1}$, case without $\mathrm{H}_{2} \mathrm{O}$-electron collisions.

(Fig. 5a), as anticipated. Nevertheless, the further relaxation of levels populations is similar to the previous case (i.e. appears to be somewhat longer in the MC method). This is due to a more accurate treatment of the radiation coming from the inner region and supporting the induced transitions.

The value of the velocity gradient is important for application of the EP method. In the case of spherical expansion it is scaled by the ratio $v_{\text {exp }} / v_{\text {th }}$. We performed calculations (not presented here) with various ratios $v_{\text {exp }} / v_{\text {th }}$ (from 1.2 up to 7 ), covering all the range of possible $v_{\exp }$ in cometary comas. Results qualitatively similar to the presented case $\left(v_{\text {exp }} / v_{\text {th }} \sim 4\right)$ are obtained when increasing $v_{\text {exp }} / v_{\text {th }}$ up to 7 . The influence of the highly excited region only decreases at distances below $R_{\mathrm{cs}}$.

For small $v_{\text {exp }} / v_{\text {th }}$ ratios the EP method overestimates level populations and the region of relaxation is enlarged. Beyond $R_{\mathrm{cs}}$ the relative differences in level populations are $\delta n / n \sim 0.25-0.3$ in the intermediate region between thermal and fluorescence equilibrium. Nonetheless we should keep in mind that such a low value of $v_{\exp } / v_{\text {th }}(1.2)$ is unrealistic in cometary atmospheres.

\subsection{Line profiles}

For practical purposes, it is important to know how the emergent line profiles (the synthetic line profiles) depend on the method used for the calculation of the excitation state. Having computed the distributions of level populations by the MC and the EP methods, we know the source function for a grid of points in the coma. The procedure of line profiles calculation requires an integration of the emergent intensities at different frequencies over a grid of lines of sight. Since we use the same code for level populations obtained by the MC and the EP methods, differences in line shapes are the consequence of different source functions only.

In order to make a link with real observations, we have chosen parameters corresponding to the capabilities of the Herschel Space Observatory and Odin. We assumed Gaussian beams and investigated Earth-comet distances $\Delta$ from 1 to $0.1 \mathrm{AU}$. In Table 4 are listed the lines investigated and the corresponding Full Width to Half Maximum (FWHM) of Herschel and Odin beams. Spatial integration covers $2.5 F W H M$. The velocity resolution for our simulations is $100 \mathrm{~m} \mathrm{~s}^{-1}$.

Figures 6 and 7 show synthetic line profiles in main beam brightness temperature scale $\left(T_{\mathrm{mB}}\right)$ for the $1_{10}-1_{01}, 2_{12}-1_{01}$, $3_{03}-2_{12}$ and $3_{12}-3_{03}$ transitions that will be observable with HIFI. The $1_{10}-1_{01}$ line is the only water line observable with Odin. Figure 8 shows a synthetic profile of this line for Odin beam. Line profiles obtained for $\Delta=0.1 \mathrm{AU}$ and
$Q_{\mathrm{H}_{2} \mathrm{O}}=10^{29} \mathrm{~s}^{-1}$ are shown in Fig. 9. In all cases and for all lines, the relative differences in brightness temperature $\delta T_{\mathrm{mB}} / T_{\mathrm{mB}} \leq$ 0.06 . The line width of the line and positions of the maximums are also in excellent agreement.

We also studied the sensitivity of the relative difference of the emerging intensity (i.e. line area $I=\int T_{\mathrm{mB}} \mathrm{d} v$ ) of the lines $\delta I / I$ to the field of view. As an example $\delta I / I$ is shown in Fig. 10 for the case $Q_{\mathrm{H}_{2} \mathrm{O}}=10^{29} \mathrm{~s}^{-1}$ (with and without $\mathrm{H}_{2} \mathrm{O}$-electron collisions) as a function of the field of view radius $\left(R_{\text {fov }}\right)$. One can see that the maximal values do not exceed 0.07 even in the case when $\mathrm{H}_{2} \mathrm{O}$-electron collisions are considered. In Fig. 10a, the first maximum at $R_{\mathrm{fov}} \sim R_{\mathrm{cs}} / 2$ is caused by the differences in level populations in the narrow region with strong $T_{\mathrm{e}}$ and $n_{\mathrm{e}}$ gradients. A second maximum is present at $R_{\text {fov }}$ equal to about half the distance where fluorescence equilibrium starts. As expected, in the case without $\mathrm{H}_{2} \mathrm{O}$-electron collisions, the first maximum is not present (Fig. 10b).

Table 4 lists line areas at $\Delta=1$ AU obtained with the MC method. Line velocity shifts with respect to comet rest velocity are also given. Most lines are redshifted as a result of self-absorption effects (Figs. 6-9). This was observed on real cometary spectra (Lecacheux et al. 2003; Biver et al. 2007). Note that, in real cases, anisotropic outgassing also affects the line shape.

\section{Conclusions}

Application of the Monte Carlo and escape probability methods for the simulation of ortho-water rotational excitation in spherically symmetric cometary atmospheres with constant velocity has shown that:

1. Level populations computed by the two methods are in reasonable agreement $(\delta n / n<0.15)$ for most parts of the coma. Strong differences $(\delta n / n \approx 2-4)$ are present only in the vicinity of the region with strong gradients of $T_{\mathrm{e}}$ and $n_{\mathrm{e}}$. When this strong gradient is absent (i.e., when $\mathrm{H}_{2} \mathrm{O}$-electron collisions are removed) $\delta n / n<0.1$ everywhere in the coma. This holds throughout the range of possible values of $v_{\text {exp }} / v_{\text {th }}$.

The discrepancies between the two methods can be considered acceptable, since the uncertainty in model parameters leads to much greater deviations. For instance, variation of the scaling factor $x_{n_{\mathrm{e}}}$ from 1 to 0.2 changes the populations by 10 to $80 \%$ in the intermediate region between thermal and fluorescence equilibrium. 
Table 4. Expected line intensities and velocity shifts at $\Delta=1 \mathrm{AU}$ computed with the MC method.

\begin{tabular}{|c|c|c|c|c|c|c|c|}
\hline \multirow[t]{2}{*}{ Telescope } & \multirow[t]{2}{*}{ Line } & \multirow[t]{2}{*}{$v_{i j}[\mathrm{GHz}]$} & \multirow[t]{2}{*}{$F W H M[\operatorname{arcsec}]$} & \multicolumn{2}{|c|}{$\int T_{\mathrm{mB}} \mathrm{d} v\left[\mathrm{~K} \mathrm{~km} \mathrm{~s}^{-1}\right]$} & \multicolumn{2}{|c|}{$\Delta v\left[\mathrm{~km} \mathrm{~s}^{-1}\right]$} \\
\hline & & & & $10^{28 \dagger}$ & $10^{29 \dagger}$ & $10^{28 \dagger}$ & $10^{29 \dagger}$ \\
\hline Odin & $1_{10}-1_{01}$ & 556.936 & 126.0 & 0.69 & 5.88 & 0.059 & 0.14 \\
\hline Herschel & $1_{10}-1_{01}$ & 556.936 & 39.0 & 3.63 & 25.85 & 0.11 & 0.22 \\
\hline Herschel & $2_{12}-1_{01}$ & 1669.904 & 13.0 & 5.44 & 43.75 & 0.20 & 0.31 \\
\hline Herschel & $2_{21}-1_{10}$ & $2773.977^{*}$ & 7.8 & 2.15 & 21.04 & 0.18 & 0.30 \\
\hline Herschel & $2_{21}-2_{12}$ & 1661.007 & 13.1 & 1.01 & 16.22 & 0.056 & 0.13 \\
\hline Herschel & $3_{03}-2_{12}$ & 1716.770 & 12.7 & 2.43 & 24.35 & 0.038 & 0.14 \\
\hline Herschel & $3_{12}-2_{21}$ & 1153.127 & 18.8 & 0.14 & 4.01 & 0.00018 & 0.00031 \\
\hline Herschel & $3_{12}-3_{03}$ & 1097.365 & 19.8 & 0.65 & 11.11 & 0.0058 & 0.021 \\
\hline Herschel & $3_{21}-2_{12}$ & $3977.047^{*}$ & 5.5 & 0.68 & 7.03 & 0.10 & 0.22 \\
\hline Herschel & $3_{21}-3_{12}$ & 1162.912 & 18.7 & 0.15 & 4.04 & 0.014 & 0.029 \\
\hline
\end{tabular}

* Observable with PACS.

${ }^{\dagger}$ Water production rate $Q_{\mathrm{H}_{2} \mathrm{O}}\left[\mathrm{s}^{-1}\right]$.
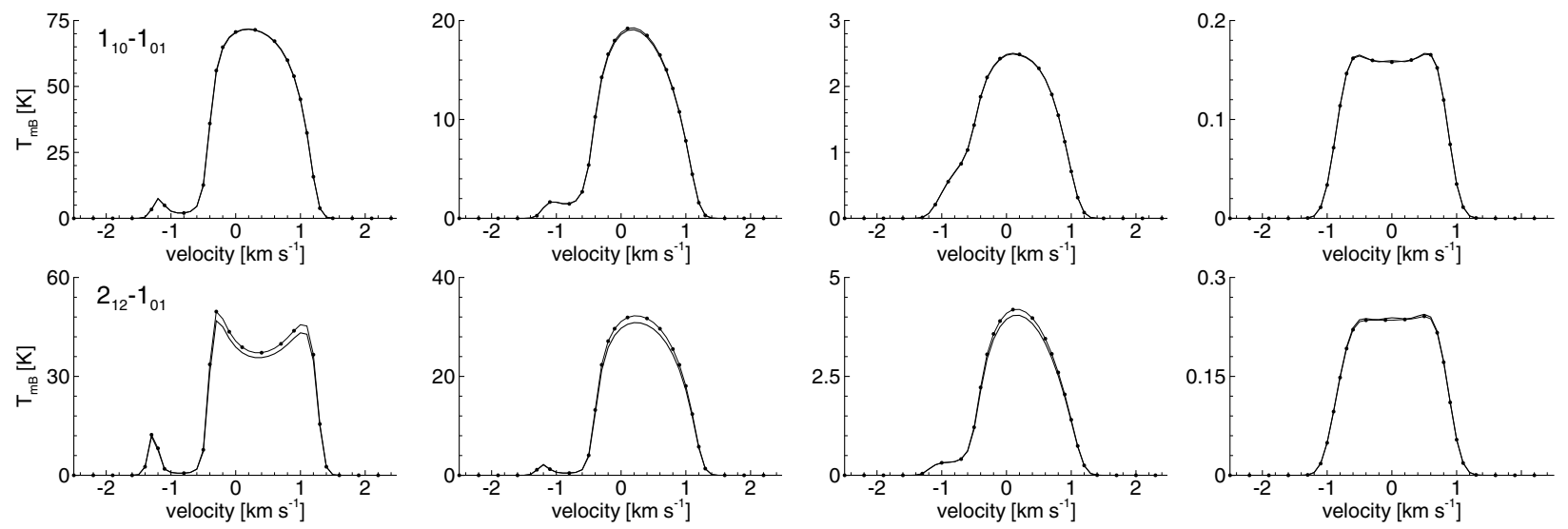

Fig. 6. Synthetic profiles for $1_{10}-1_{01}$ and $2_{12}-1_{01}$ lines observable with HIFI for $\Delta=1$ AU. From left to right: $Q_{\mathrm{H}_{2} \mathrm{O}}=10^{30}, 10^{29}, 10^{28} \mathrm{~s}^{-1}$ and $10^{29} \mathrm{~s}^{-1}$ for $\mathrm{H}_{2}^{18} \mathrm{O}$ (MC - thin lines and plain symbols, $\mathrm{EP}$ - thick lines).
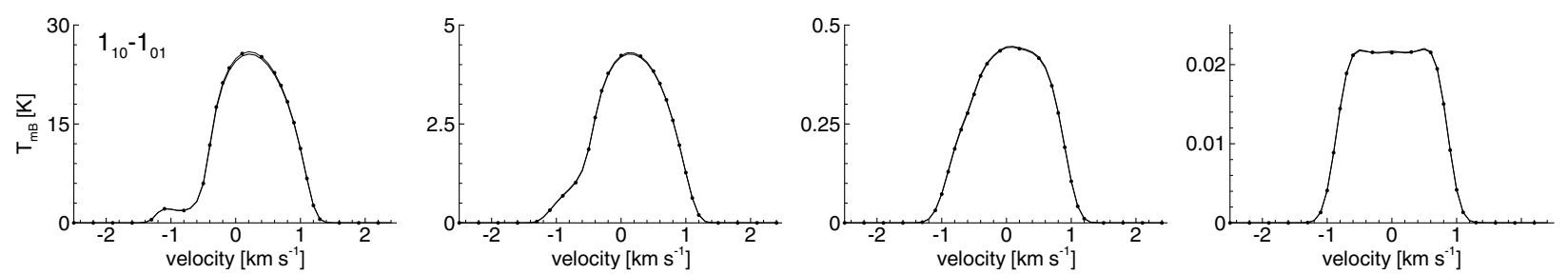

Fig. 8. Synthetic profiles for $1_{10}-1_{01}$ line observed with Odin for $\Delta=1$ AU. From left to right: $Q_{\mathrm{H}_{2} \mathrm{O}}=10^{30}, 10^{29}, 10^{28} \mathrm{~s}^{-1}$ and $10^{29} \mathrm{~s}^{-1}$ for $\mathrm{H}_{2}^{18} \mathrm{O}$ (MC - thin lines and plain symbols, EP - thick lines).
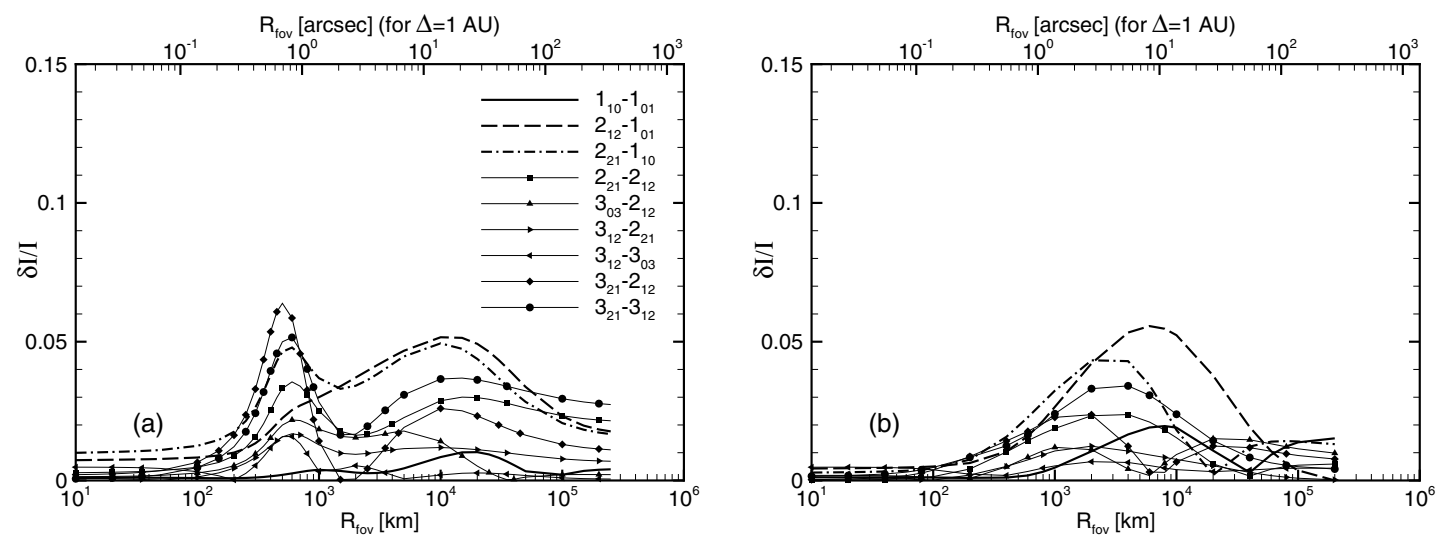

Fig. 10. Relative differences of line areas $\delta I / I$ as a function of beam radius $\left(R_{\text {fov }}\right)$ for a) $Q_{\mathrm{H}_{2} \mathrm{O}}=10^{29} \mathrm{~s}^{-1}$, b) same without electrons. The upper scale shows the beam radius in arcsec for a comet at $\Delta=1 \mathrm{AU}$. 
2. Synthetic line profiles derived from the source functions obtained by the MC and the EP methods have the same shapes and their $\delta T_{\mathrm{mB}} / T_{\mathrm{mB}} \leq 0.06$ for the $557 \mathrm{GHz}$ line observed with Odin and for the water lines to be observed with the HIFI/Herschel instrument.

The CPU time needed for the Monte Carlo computations (tens of hours with an AMD Opteron $800 \mathrm{MHz}$ processor for the computations presented here) is much longer than that needed with the EP method (a few seconds).

Thus we can conclude that the EP method is a reasonable and convenient approach for treating radiation transfer and line excitation of water (and of other molecules with thick rotational lines) in cometary atmospheres. In most observational cases, errors introduced by the use of the EP method will be less than those resulting from uncertainties in model parameters such as gas temperature, or electron density and temperature radial profiles. Previous analyses of cometary $\mathrm{H}_{2} \mathrm{O} 557 \mathrm{GHz}$ data that made use of the EP method (e.g., Lecacheux et al. 2003; Biver et al. 2007, for the Odin data; Chiu et al. 2001, for the SWAS data) are thus validated.

Bensch \& Bergin (2004, 2007) compare water production rates determined from SWAS data in C/1999 T1 (McNaught-Hartley) with either their MC code or our code which uses the EP method (Bensch et al. 2004). They find a $20-40 \%$ discrepancy that they attribute to the different numerical methods used for solving radiative transfer. The results presented in the present paper do not agree with their conclusion. The discrepancy is likely related to differences in model input parameters.

We studied here the case of spherically symmetric comas with constant gas velocity. Real radio cometary lines present asymmetries in line shape which can be attributed to the anisotropy of the coma. In addition, any realistic coma presents radial and azimutal variations in gas velocity. To study in detail such cases, the Monte Carlo approach will be mandatory. Given the computational demands of this approach, methods inspired from the 3D multi-zone escape probability method (Poelman \& Spaans 2005) or the Coupled Escape Probability (CEP) method (Elitzur \& Asensio Ramos 2006) could be excellent alternatives.
Acknowledgements. This work was made possible thanks to the support of the French space agency (Centre National d'Etudes Spatiales), in the frame of the Odin space mission.

\section{References}

Bastian, U., Bertout, C., Stenholm, L., \& Wehrse, R. 1980, A\&A, 86, 105 Bensch, F., \& Bergin, E. A. 2004, ApJ, 615, 531

Bensch, F., \& Bergin, E. A. 2007, ApJ, 659, 1795 (Erratum)

Bensch, F., Bergin, E. A., Bockelée-Morvan, D., Melnick, G. J., \& Biver, N. 2004, ApJ, 609, 1164

Bernes, C. 1979, A\&A, 73, 67

Biver, N. 1997, Ph.D. Thesis, Univ. Paris VII

Biver, N., Bockelée-Morvan, D., Crovisier, J., et al. 2007, Planet. Space Sci., 55, 1058

Bockelée-Morvan, D. 1987, A\&A, 181, 169

Bockelée-Morvan, D., \& Crovisier, J. 1989, A\&A, 216, 278

Bockelée-Morvan, D., Crovisier, J., Mumma, M. J., \& Weaver, H. A. 2004, in Comets II, ed. M. C. Festou, H. U. Keller, \& H. A. Weaver (The University of Arizona Press), 391

Buffa, G., Tarrini, O., Scappini, F., \& Cecchi-Pestellini, C. 2000, ApJS, 128, 597

Chiu, K., Neufeld, D. A., Bergin, E. A., et al. 2001, Icarus, 154, 345

Crovisier, J. 1984, A\&A, 135, 197

Crovisier, J. 2005, in The dusty and molecular universe: a prelude to Herschel and ALMA, ed. A. Wilson, ESA SP-577, 14

Crovisier, J., Leech, K., Bockelée-Morvan, D., et al. 1997, Science, 275, 1904

Elitzur, M., \& Asensio Ramos, A. 2006, MNRAS, 365, 779

Feldman, P. D., Cochran, A. L., \& Combi, M. R. 2004, In Comets II, ed. M. C. Festou, H. U. Keller, \& H. A. Weaver (The University of Arizona Press), 425

de Graauw, Th., \& Helmich, F. P. 2001, in The Promise of the Herschel Space Observatory, ed. G. L. Pilbratt, et al. ESA-SP 460, 45

Gulkis, S., Frerking, M., Crovisier, J., et al. 2007, Space Sci. Rev., 128, 561

Haser, L. 1957, Bull. Acad. R. Sci. Liège, 43, 740

Hogerheijde, M. R., \& van der Tak, F. F. S. 2000, A\&A, 362, 697

Itikawa, Y. 1972, J. Phys. Soc. Japan, 32, 217

Jacquinet-Husson, N., Arié, E., Ballard, J., et al. 1999, J. Quant. Spec. Radiat. Transf., 62, 205

Lecacheux, A., Biver, N., Crovisier, J., et al. 2003, A\&A, 402, L55

Litvak, M. M., \& Kuiper, E. N. R. 1982, ApJ, 253, 622

Melnick, G. J., Stauffer, J. R., Ashby, M. L. N., et al. 2000, ApJ, 539, L77

Neufeld, D. A., Stauffer, J. R., Bergin, E. A., et al. 2000, ApJ, 539, L151

Nordh, H. L., von Scheele, F., Frisk, U., et al. 2003, A\&A, 402, L21

Pilbratt, G. L. 2005, in The dusty and molecular universe: a prelude to Herschel and ALMA, ed. A. Wilson, ESA SP-577, 3

Poelman, D. R., \& Spaans, M. 2005, A\&A, 440, 559

Rybicki, G. B. 1984, in Methods of Radiative Transfer, ed. W. Kalkofen (Cambridge: Cambridge University Press), 21

Weaver, H. A., \& Mumma, M. J. 1984, ApJ, 276, 782

Xie, X., \& Mumma, M. J. 1992, ApJ, 386, 720 


\section{Online Material}


V. Zakharov et al.: Radiative transfer for water in comets, Online Material p 2

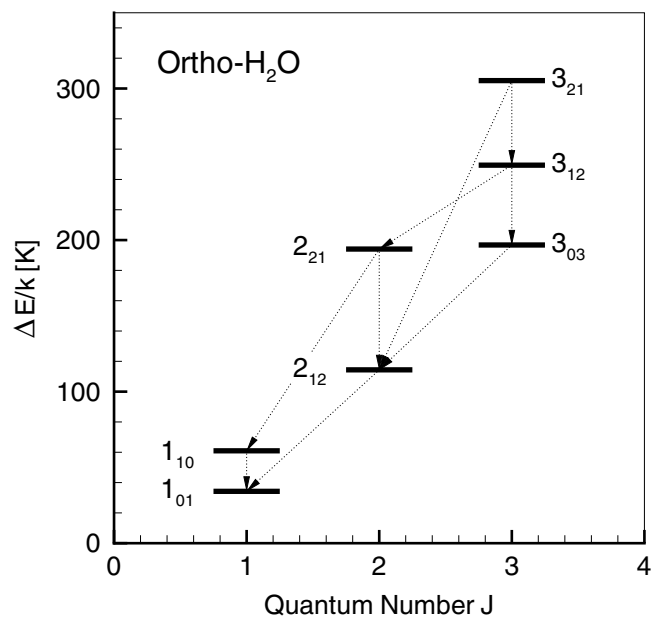

Fig. 1. $\mathrm{H}_{2} \mathrm{O}$ rotational levels and lines considered in the models.

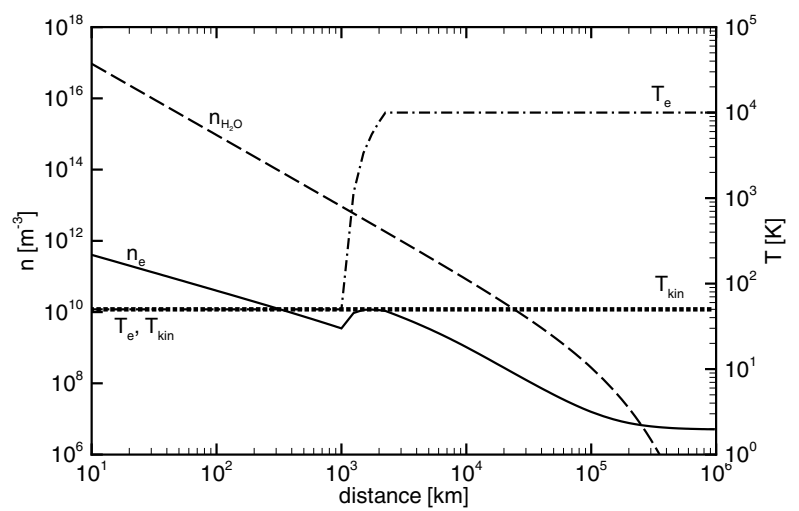

Fig. 2. Evolution of $\mathrm{H}_{2} \mathrm{O}$ and electron density and temperature with distance to nucleus for $Q_{\mathrm{H}_{2} \mathrm{O}}=10^{29} \mathrm{~s}^{-1}$ and the parameters given in Table 1. 
V. Zakharov et al.: Radiative transfer for water in comets, Online Material $p 3$

Table 3. Einstein A-coefficients for spontaneous emission $A_{i j}\left[\mathrm{~s}^{-1}\right], \mathrm{H}_{2} \mathrm{O}-\mathrm{H}_{2} \mathrm{O}$ collisional cross-section for de-excitation $\sigma_{i j}$ [m ${ }^{2}$, "effective" pumping rates $G_{i j}\left[\mathrm{~s}^{-1}\right]$, and line central frequencies $v_{i j}[\mathrm{GHz}]$.

\begin{tabular}{|c|c|c|c|c|c|c|c|c|}
\hline$\overline{i \backslash j}$ & & $1_{01}$ & $\overline{1_{10}}$ & $\overline{22_{12}}$ & $22_{21}$ & $3_{03}$ & $\overline{3}_{12}$ & $3_{21}$ \\
\hline $1_{01}$ & $\begin{array}{l}A_{i j} \\
\sigma_{i j} \\
G_{i j} \\
v_{i j} \\
\end{array}$ & & $1.654 \times 10^{-5}$ & $2.464 \times 10^{-5}$ & $8.486 \times 10^{-5}$ & $1.471 \times 10^{-4}$ & $1.359 \times 10^{-5}$ & $2.905 \times 10^{-5}$ \\
\hline $1_{10}$ & $\begin{array}{l}A_{i j} \\
\sigma_{i j} \\
G_{i j} \\
v_{i j} \\
\end{array}$ & $\begin{array}{c}3.456 \times 10^{-3} \\
2.924 \times 10^{-18} \\
1.423 \times 10^{-5} \\
556.936 \\
\end{array}$ & & $1.882 \times 10^{-4}$ & $1.696 \times 10^{-5}$ & $1.118 \times 10^{-5}$ & $9.143 \times 10^{-5}$ & $1.294 \times 10^{-5}$ \\
\hline $2_{12}$ & $\begin{array}{l}A_{i j} \\
\sigma_{i j} \\
G_{i j} \\
v_{i j} \\
\end{array}$ & $\begin{array}{c}5.588 \times 10^{-2} \\
3.248 \times 10^{-18} \\
1.323 \times 10^{-5} \\
1669.904 \\
\end{array}$ & $1.154 \times 10^{-4}$ & & $1.315 \times 10^{-5}$ & $1.492 \times 10^{-5}$ & $1.341 \times 10^{-4}$ & $1.421 \times 10^{-5}$ \\
\hline $2_{21}$ & $\begin{array}{l}A_{i j} \\
\sigma_{i j} \\
G_{i j} \\
v_{i j}\end{array}$ & $5.619 \times 10^{-5}$ & $\begin{array}{c}2.572 \times 10^{-1} \\
2.162 \times 10^{-18} \\
1.602 \times 10^{-5} \\
2773.977 \\
\end{array}$ & $\begin{array}{c}3.054 \times 10^{-2} \\
2.327 \times 10^{-18} \\
1.531 \times 10^{-5} \\
1661.007 \\
\end{array}$ & & $2.846 \times 10^{-5}$ & $1.694 \times 10^{-5}$ & $1.415 \times 10^{-4}$ \\
\hline $3_{03}$ & $\begin{array}{l}A_{i j} \\
\sigma_{i j} \\
G_{i j} \\
v_{i j} \\
\end{array}$ & $6.620 \times 10^{-5}$ & $5.189 \times 10^{-6}$ & $\begin{array}{c}5.051 \times 10^{-2} \\
2.627 \times 10^{-18} \\
1.113 \times 10^{-5} \\
1716.770 \\
\end{array}$ & $1.982 \times 10^{-5}$ & & $1.013 \times 10^{-5}$ & $5.302 \times 10^{-5}$ \\
\hline $3_{12}$ & $\begin{array}{l}A_{i j} \\
\sigma_{i j} \\
G_{i j} \\
v_{i j} \\
\end{array}$ & $4.428 \times 10^{-6}$ & $4.090 \times 10^{-5}$ & $1.006 \times 10^{-4}$ & $\begin{array}{c}2.659 \times 10^{-3} \\
6.840 \times 10^{-19} \\
5.843 \times 10^{-6} \\
1153.127 \\
\end{array}$ & $\begin{array}{c}1.641 \times 10^{-2} \\
2.204 \times 10^{-18} \\
7.287 \times 10^{-6} \\
1097.365 \\
\end{array}$ & & $9.177 \times 10^{-6}$ \\
\hline $3_{21}$ & $\begin{array}{l}A_{i j} \\
\sigma_{i j} \\
G_{i j} \\
v_{i j} \\
\end{array}$ & $1.448 \times 10^{-5}$ & $7.867 \times 10^{-6}$ & $\begin{array}{c}3.325 \times 10^{-1} \\
6.570 \times 10^{-19} \\
9.250 \times 10^{-6} \\
3977.047 \\
\end{array}$ & $1.038 \times 10^{-4}$ & $5.593 \times 10^{-5}$ & $\begin{array}{c}2.280 \times 10^{-2} \\
2.322 \times 10^{-18} \\
1.166 \times 10^{-5} \\
1162.912 \\
\end{array}$ & \\
\hline
\end{tabular}
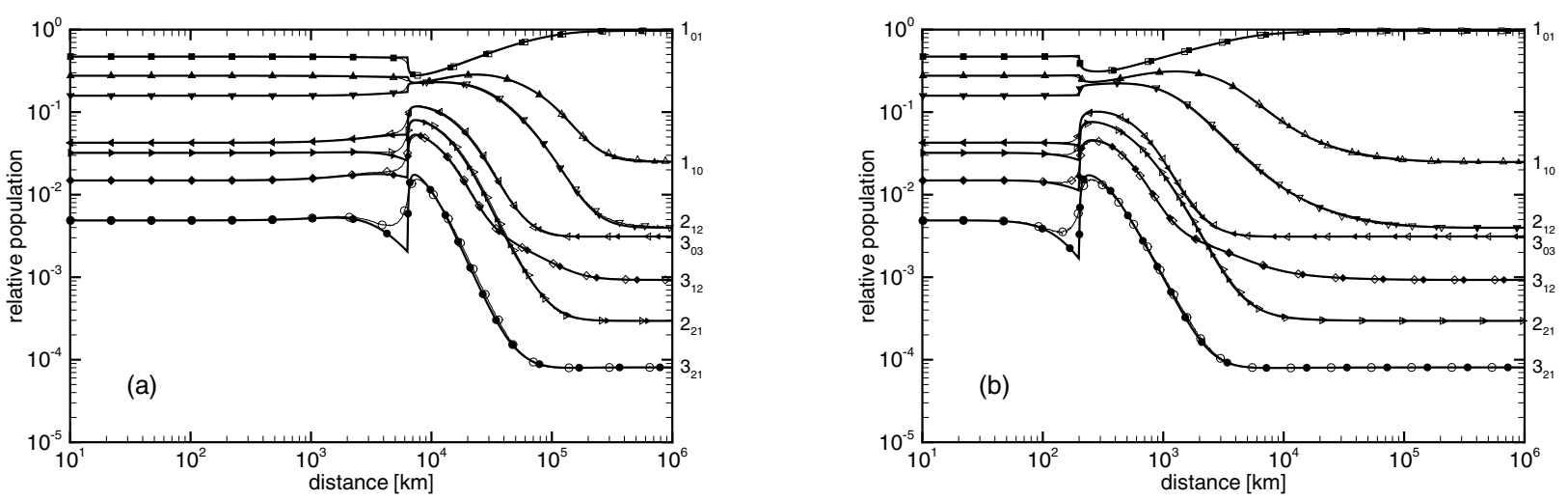

Fig. 4. Level populations of $\mathrm{H}_{2} \mathrm{O}$ by the $\mathrm{MC}$ (thin lines and open symbols) and the EP (thick lines and plain symbols) methods a) for $Q_{\mathrm{H}_{2} \mathrm{O}}=10^{30}$ and b) $10^{28} \mathrm{~s}^{-1}$. 
V. Zakharov et al.: Radiative transfer for water in comets, Online Material p 4
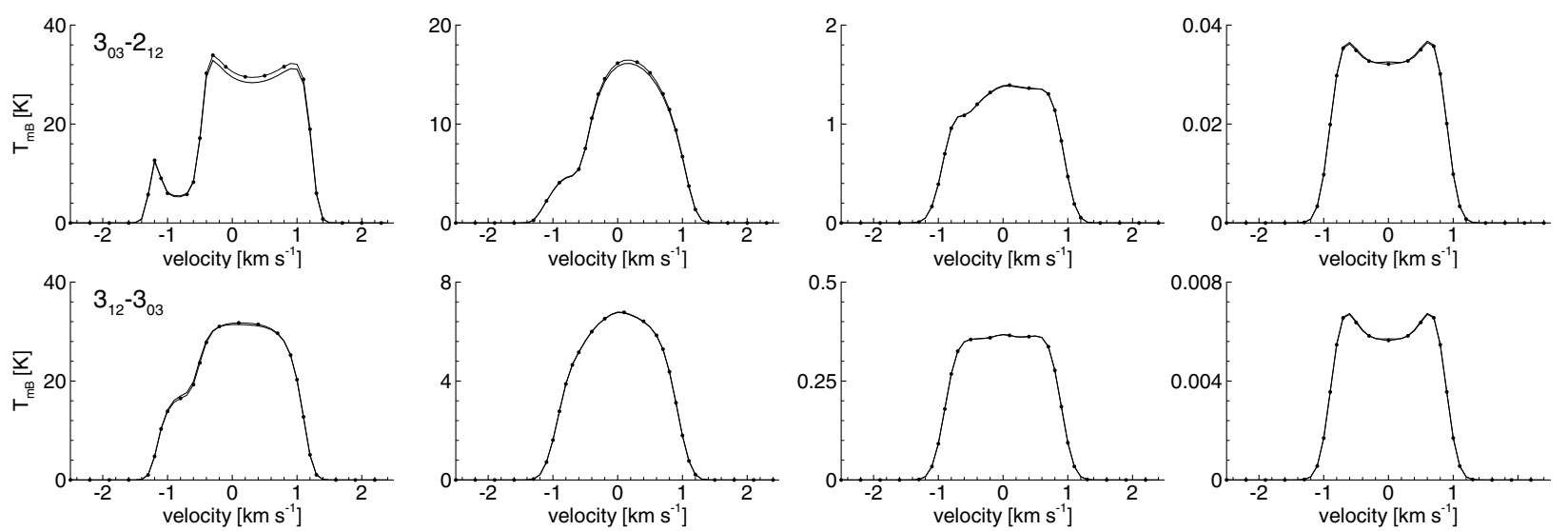

Fig. 7. Synthetic profiles for $3_{03}-2_{12}$ and $3_{12}-3_{03}$ lines observable with HIFI for $\Delta=1$ AU. From left to right: $Q_{\mathrm{H}_{2} \mathrm{O}}=10^{30}, 10^{29}, 10^{28} \mathrm{~s}^{-1}$ and $10^{29} \mathrm{~s}^{-1}$ for $\mathrm{H}_{2}^{18} \mathrm{O}(\mathrm{MC}-$ thin lines and plain symbols, $\mathrm{EP}$ - thick lines).
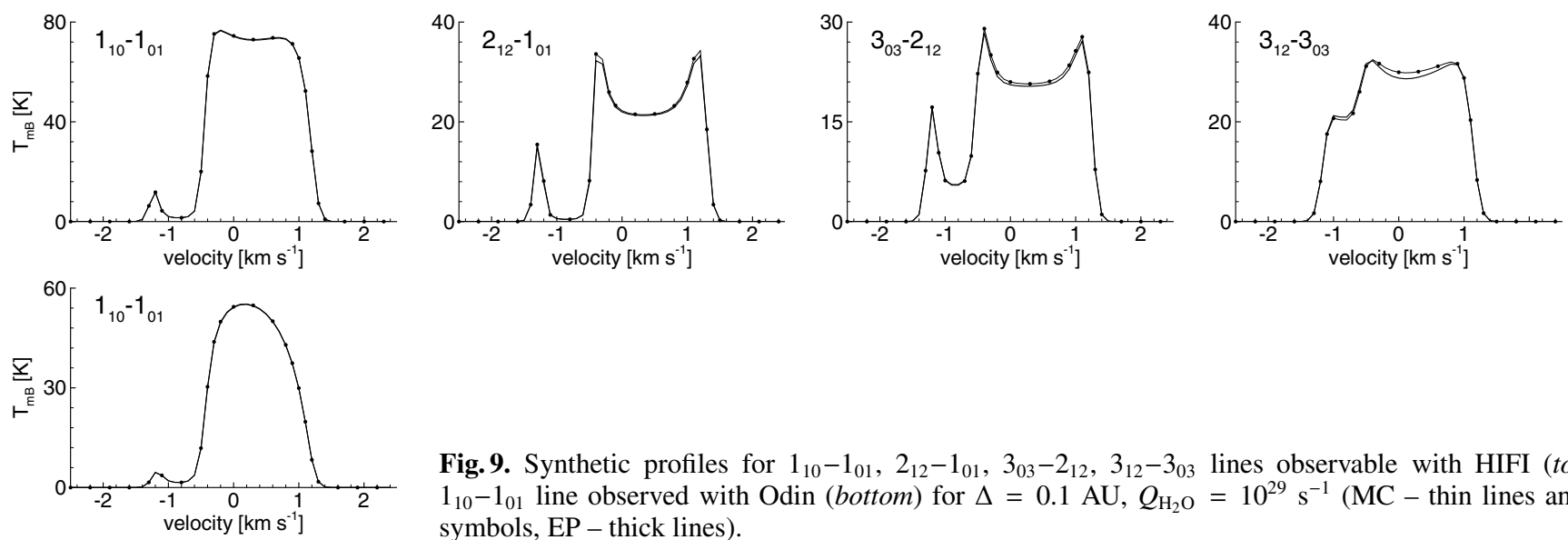

Fig. 9. Synthetic profiles for $1_{10}-1_{01}, 2_{12}-1_{01}, 3_{03}-2_{12}, 3_{12}-3_{03}$ lines observable with HIFI (top) and $1_{10}-1_{01}$ line observed with Odin (bottom) for $\Delta=0.1 \mathrm{AU}, Q_{\mathrm{H}_{2} \mathrm{O}}=10^{29} \mathrm{~s}^{-1}$ ( $\mathrm{MC}$ - thin lines and plain symbols, EP - thick lines). 